\title{
Optimisation de la décantation des rejets urbains de temps de pluie dans les bassins de retenue contre les inondations
}

\author{
par Chantal Jacopin, Jean-Luc Bertrand-Krajewski \\ GARIH/CTIA Lyonnaise des Eaux
}

Michel Desbordes

ISIM, Université de Montpellier

Pierre Bourgogne

Communauté Urbaine de Bordeaux

\section{I $\square$ CONTEXTE DE L'ÉTUDE}

\begin{abstract}
Apparus en France il y a moins d'une trentaine d'années, les bassins de retenue constituent un moyen efficace pour remédier aux insuffisances hydrauliques des réseaux d'assainissement unitaires et séparatifs [1]. Leur volume spécifique est généralement élevé : de l'ordre de 300 à $600 \mathrm{~m}^{3} / \mathrm{ha}$ imperméabilisé pour la protection décennale dans le cas de Bor-
\end{abstract}

deaux et, dans ces conditions, les temps de séjour des eaux stockées sont suffisamment longs (une à plus de deux heures) pour qu'une décantation des solides en suspension s'opère et conduise à une dépollution non négligeable des eaux.

Jusqu'à présent, la décantation s'est produite de façon non contrôlée ; aujourd'hui au vu des rendements déjà obtenus, on cherche à améliorer l'exploitation des bassins de retenue pour l'optimiser.

Detention tanks in combined or separate sewer systems have been used since the last three decades in order to reduce the floods due to the urban runoff. In addition to their hydraulic efficiency, it appears that they reduce the polluant discharges by settling. The main objective of our study is the optimisation of the settling process in this type of tank by the improvement of the operational rules. The first stage of the study is the characterization of the suspended solids and of their settling process in a combined underground tank and in separate detention tank with grassy bottom.

The grain size of sediments sampled in the combined tank and solids from the superficial layer $(0-10 \mathrm{~cm})$ of the separate tank is fine and close to the grain size distribution of suspended solids in stormwater discharges. A grain size segregation of the solids is observed : the filling and emptying process and the tank geometry influence their distribution. For the tank with grassy bottom, the flooding frequency is also a very important parameter. The pollutant concentrations (heavy metals and hydrocarbons) are correlated with the grain size distribution: the finer the solids are, the more polluting they are. These concentrations are high in the combined tank and in the part of the separate tank where the settling process is frequent. On the other hand, in the separate tank, the pollutant concentrations of the layers $10-30 \mathrm{~cm}$ and $30-50 \mathrm{~cm}$ are light and decrease with depth up to values corresponding to unpolluted natural soils. 


\section{II — OBJECTIFS DE L'ÉTUDE}

Initiée par le Groupement Aquitain pour la Recherche et I'Innovation en Hydrologie (GARIH), une étude de trois ans portant sur ce thème de recherche a débuté à Bordeaux en mai 1996.

Son objectif principal est d'optimiser la décantation naturelle des solides en suspension dans les Rejets urbains de temps de pluie dans les bassins de retenue conçus initialement pour des raisons hydrauliques, la fonction de lutte contre les polluants complétant alors, pour les pluies de faible ou moyenne importance, la fonction de lutte contre les inondations de ces bassins. Actuellement, pour limiter les risques d'inondations, les bassins sont vidangés aussi rapidement que possible après leur remplissage, dès que les conditions hydrauliques aval dans le réseau le permettent. Aussi, si l'on cherche à favoriser la décantation des rejets urbains de temps de pluie dans ces bassins, cette gestion devra-t-elle être modifiée : il faudra conserver pendant plusieurs heures les eaux stockées afin d'obtenir une décantation maximale des matières en suspension.

Le travail de recherche comporte plusieurs phases:

- étude de la décantation des matières en suspension dans les bassins : vitesse de chute, granulométrie, charges polluantes, description hydrodynamique ;

- analyse et modélisation du fonctionnement actuel des bassins, grâce à une analyse statistique des données de régulation enregistrées depuis 1992 par le télécontrôle RAMSES ; - simulation de nouvelles règles de fonctionnement et de gestion des bassins optimisant la décantation et analyse statistique de l'accroissement éventuel du risque d'inondation lié à l'optimisation de la décantation.

Cet article aborde essentiellement les résultats déjà obtenus sur la caractérisation et la décantation dans les bassins de retenue des solides véhiculés dans les RUTP.

\section{III — SITES ET MATÉRIELS}

Pour mener cette étude, deux bassins de retenue expérimentaux ont été sélectionnés à Bordeaux (tabl. I).

Sur chacun des sites, nous disposons de l'équipement suivant en amont et en aval des bassins : débitmètre, station de mesure en continu ( $\mathrm{pH}$, conductivité, température, turbidité), échantillonneur (fig. I).

Pour compléter et affiner les résultats du suivi en continu des événements pluvieux, des campagnes ponctuelles de prélèvement d'eaux pluviales et de dépôts sont réalisées régulièrement et des pièges à sédiments ont été installés dans le fond des bassins.

\section{IV — RÉSULTATS DES PREMIÈRES CAM- PAGNES D'ÉTUDE DE LA DÉCANTA- TION}

\subsection{Bassin de Périnot}

Des campagnes de prélèvements de dépôts en place ont pour objet de déterminer la variabilité et les caractéristiques moyennes des solides décantés au fil des remplissages par temps de pluie. Les dépôts récupérés dans les pièges à sédiments permettent de déterminer les caractéristiques des solides propres à un événement pluvieux.

\subsubsection{Granulométrie}

Les solides formant les dépôts du bassin de Périnot sont d'une granulométrie plutôt fine se rapprochant de la granulométrie moyenne des particules en suspension dans les RUTP (Tab. 2). Mais une analyse plus précise des résultats montre

Tableau 1. - Caractéristiques des sites expérimentaux de Périnot et du Bourgailh.

\begin{tabular}{|c|c|c|c|c|c|c|}
\hline \multirow{2}{*}{$\begin{array}{l}\text { Bassins } \\
\text { de retenue } \\
\text { étudiés }\end{array}$} & \multicolumn{3}{|c|}{ Caractéristiques du bassin versant } & \multicolumn{3}{|c|}{ Caractéristiques du bassin } \\
\hline & Réseau & $\begin{array}{l}\text { Taille } \\
\text { (ha) }\end{array}$ & $\begin{array}{l}\text { Imperméa- } \\
\text { bilisation(\%) }\end{array}$ & $\begin{array}{l}\text { Capacité } \\
\qquad\left(\mathrm{m}^{3)}\right.\end{array}$ & $\begin{array}{c}\text { Type de } \\
\text { Particularités }\end{array}$ & \\
\hline PERINOT & unitaire & 217 & 35 & $\begin{array}{l}38000 \\
\left(500 \mathrm{~m}^{3} / \mathrm{ha}\right. \\
\text { imp.) }\end{array}$ & $\begin{array}{c}\text { * en dérivation } \\
\text { * enterré }\end{array}$ & $\begin{array}{l}\text { Séparation en } 3 \text { compartiments } \\
\text { et remplissage par le central } \\
\text { (bas 1) }\end{array}$ \\
\hline BOURGAILH & $\begin{array}{l}\text { séparatif } \\
\text { pluvial }\end{array}$ & 676 & 18 & $\begin{array}{l}80000 \\
\left(657 \mathrm{~m}^{3} / \mathrm{ha}\right. \\
\text { imp.) }\end{array}$ & $\begin{array}{l}\text { * à ciel ouvert } \\
\text { * à sec et } \\
\text { enherbé }\end{array}$ & $\begin{array}{l}\text { Remplissage par débordement } \\
\text { du canal traversier de l'aval } \\
\text { vers l'amont }\end{array}$ \\
\hline
\end{tabular}
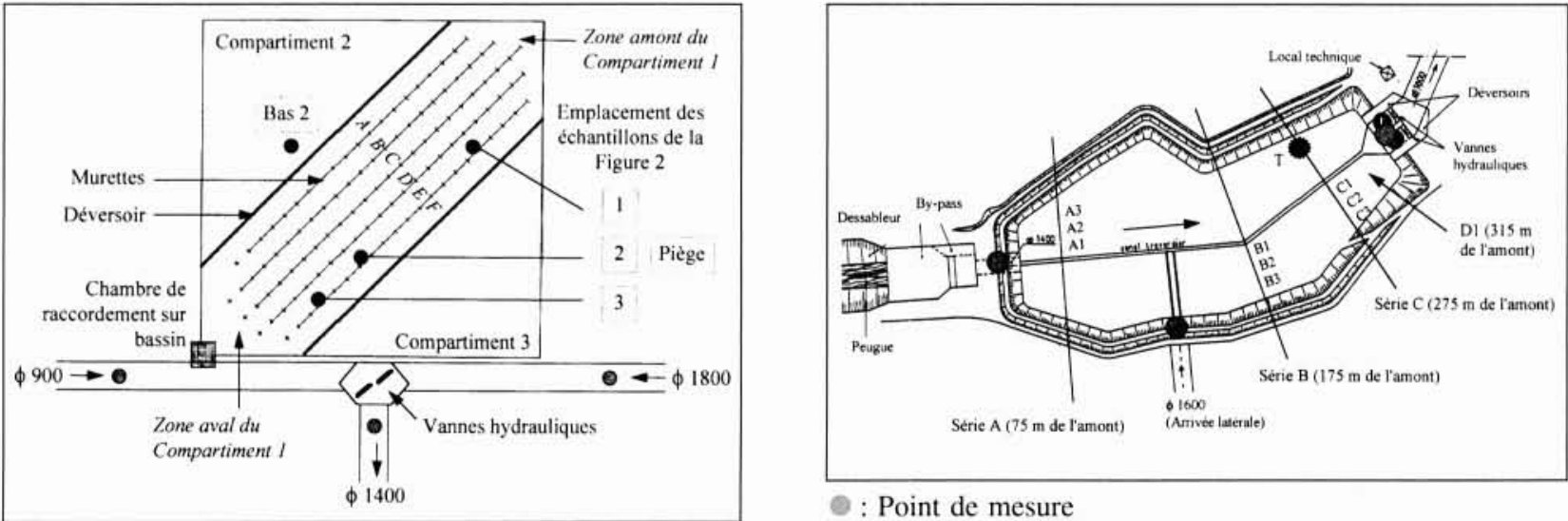

: Point de mesure

1. Bassins de retenue de Périnot (gauche) et du Bourgailh (droite). 
Tableau 2. - Granulométrie des solides décantés et des solides prélevés dans les 3 pièges à sédiments installés dans le bassin de Périnot [3].

\begin{tabular}{|l|c|c|c|c|c|c|}
\hline $\begin{array}{l}\text { Origine des } \\
\text { solides }\end{array}$ & $\begin{array}{c}\text { Date des } \\
\text { prélèvements }\end{array}$ & $\begin{array}{c}\text { Fraction }<900 \mu \mathrm{m} \\
(\%, \text { en masse })\end{array}$ & $\begin{array}{c}\text { Fraction }<100 \mu \mathrm{m} \\
(\% \text { en masse, } \\
<900 \mu \mathrm{m})\end{array}$ & $\begin{array}{c}\mathrm{d}_{10} \\
(\mu \mathrm{m})\end{array}$ & $\begin{array}{c}\mathrm{d}_{50} \\
(\mu \mathrm{m})\end{array}$ & $\begin{array}{c}\mathrm{d}_{90} \\
(\mu \mathrm{m})\end{array}$ \\
\hline $\begin{array}{l}\text { Dépôts déjà en } \\
\text { place- Bassin 1 }\end{array}$ & $\begin{array}{l}24 / 05 / 96 \text { (12 éch.) } \\
28 / 10 / 96 \text { (10 éch.) } \\
16 / 01 / 97 \text { (18 éch.) }\end{array}$ & 90,2 & 74,9 & 37,4 & 283,7 \\
\hline $\begin{array}{l}\text { Dépôts déjà en } \\
\text { place- Bassin 2 }\end{array}$ & $28 / 10 / 96$ (2 éch.) & 94,8 & 61,2 & 2,7 & 16,8 & 91,6 \\
\hline $\begin{array}{l}\text { Pièges à } \\
\text { sédiments }\end{array}$ & $\begin{array}{l}16 / 01 / 97 \text { (3 éch.) } \\
21 / 03 / 97 \text { (3 éch.) }\end{array}$ & 93,0 & 67,6 & 5,7 & 49,3 & 348,9 \\
\hline $\begin{array}{l}\text { Particules en } \\
\text { suspension dans } \\
\text { les RUTP }\end{array}$ & Réf. [2] & & 78,0 & 3,9 & 34,7 & 456,0 \\
\hline
\end{tabular}

que leur distribution granulométrique n'est pas uniforme sur l'ensemble du compartiment central. Ainsi, les dépôts prélevés dans la zone proche de l'ouvrage d'entrée-sortie (zone aval) sont plus grossiers que ceux prélevés au fond du bassin (zone amont). La même constatation s'impose lorsque l'on considère l'organisation granulométrique par travée (fig. 2).

Il semble donc que la zone aval du bassin ainsi que certaines travées (travées C, D, E) soient plus sensibles au processus de remplissage et de vidange que d'autres. La grande variabilité des volumes de dépôts d'une travée à une autre témoigne de cette sensibilité ; ils varient en effet de $16 \mathrm{~m}^{3}$ dans les travées $C$ et $\mathrm{D}$ à plus de $65 \mathrm{~m}^{3}$ dans la travée $\mathrm{E}(400$ hauteurs de dépôt mesurées en juin 1996, avant le nettoyage du bassin). Par ailleurs, en raison de la superficie du bassin, son remplissage ne peut être ni homogène ni immédiat, ce qui influe vraisemblablement sur le processus de décantation et donc sur la granulométrie des solides prélevés.

Les solides prélevés dans les 3 pièges à sédiments installés dans le compartiment central du bassin sont plus grossiers que les solides prélevés à proximité directement dans le dépôt en place.

Notons enfin les résultats granulométriques des échantillons de dépôt du compartiment 2 (figs. 1 et 2 ): les solides y sont plus fins que ceux qui constituent le dépôt du compartiment 1 . Le second compartiment du bassin se remplit en moyenne 2 fois par an (capacité $=14000 \mathrm{~m}^{3}$ ) et il reçoit alors les surverses du compartiment 1 (capacité = $10500 \mathrm{~m}^{3}$ ). La durée moyenne de remplissage de ce dernier étant d'environ 60 minutes, les surverses sont constituées par des eaux déjà décantées.

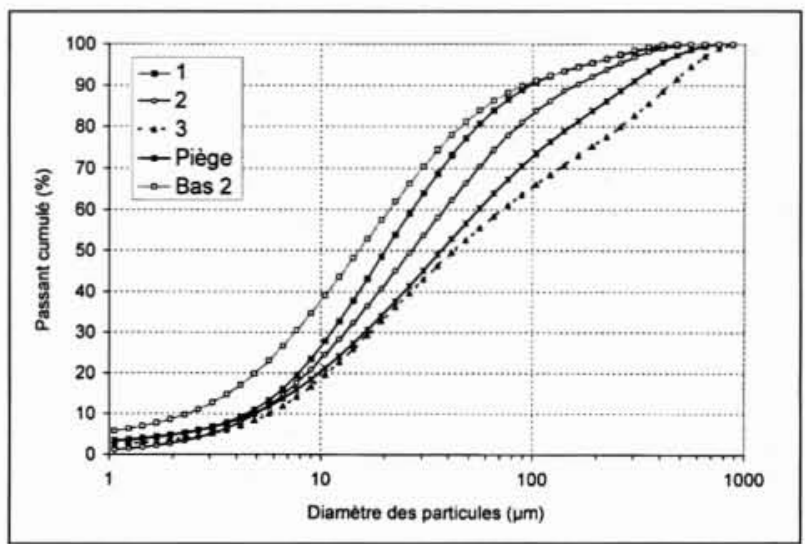

2. Courbes granulométriques de solides prélevés dans le bassin de Périnot (Campagne du 16/01/97) Cf. figure 1.

\subsubsection{Paramètres de pollution}

Les concentrations moyennes en métaux lourds et hydrocarbures des dépôts prélevés dans le bassin de Périnot sont inférieures à celles que l'on peut trouver dans la bibliographie. Mais les valeurs maximales confirment que les bassins de retenue constituent, au même titre que les dépôts de voiries et les avaloirs notamment, une source importante de pollution métallique.

Par ailleurs, les échantillons prélevés dans la zone amont sont, dans la plupart des cas, plus chargés en métaux lourds que les échantillons prélevés à proximité de l'ouvrage d'entrée-sortie (fig. 3). Nous retrouvons là un résultat déjà rencontré dans la bibliographie [4] : les particules les plus fines offrent une surface spécifique importante, ce qui favorise la fixation des métaux par adsorption sur ce type de particule. De façon opposée, nous trouvons les concentrations les plus élevées en hydrocarbures parmi les prélèvements réalisés dans la zone aval.

\subsection{Bassin du Bourgailh}

Une campagne de carottage destinée à estimer les teneurs en polluants du sol et à déceler leur migration éventuelle a été réalisée en août 1996 dans le bassin enherbé du Bourgailh.

Les emplacements des 3 séries de carottage, comportant chacune 3 prélèvements destinés à analyser les couches de sol : $0-10 \mathrm{~cm}, 10-30 \mathrm{~cm}$ et $30-50 \mathrm{~cm}$, sont indiqués sur la figure 1. D1 est un échantillon de la couche de surface $0-10 \mathrm{~cm}$ prélevé quelques semaines plus tard dans les mêmes conditions.

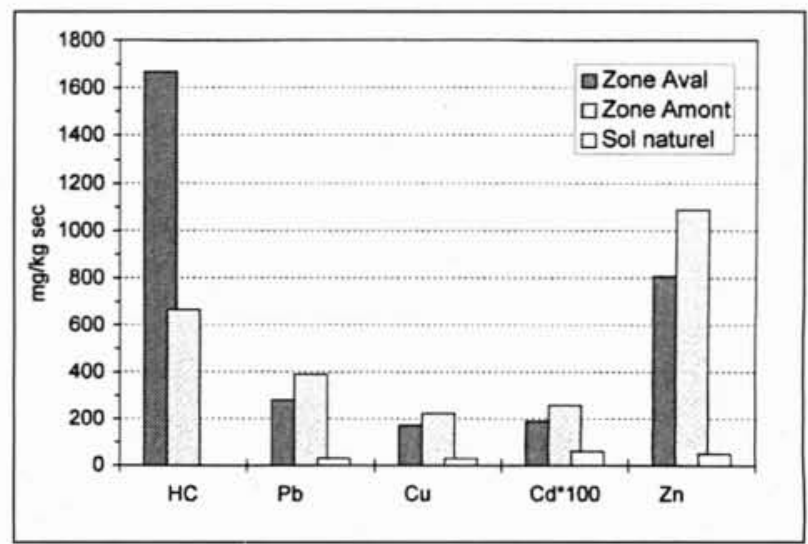

3. Concentrations moyennes en hydrocarbures et métaux lourds des prélèvements de dépôts réalisés dans le bassin de Périnot le 24/05/1996. 


\subsubsection{Granulométrie}

La couche de surface $(0-10 \mathrm{~cm})$ ainsi que les couches 10 $30 \mathrm{~cm}$ et $30-50 \mathrm{~cm}$ sont constituées essentiellement de sables et la teneur en argile est de l'ordre de $3 \%$ (tabl. 3).

Sur l'ensemble du bassin, la couche de surface présente une granulométrie plus grossière que les solides en suspension dans les RUTP ou que ceux formant les dépôts d'autres bassins de rétention, Périnot par exemple.

Cependant le diamètre des sédiments prélevés en surface diminue d'une part de l'amont vers l'aval, et d'autre part, lorsqu' on se rapproche du canal traversier (fig. 5). Par ailleurs, comme le montrent les figures 4 et 5 , il existe pour les prélèvements des séries $\mathrm{B}, \mathrm{C}$ et $\mathrm{D}$, une forte corrélation entre la fréquence de submersion des points échantillonnés et les caractéristiques granulométriques : ainsi le point D1, soumis à 41 submersions en 1996, est de granulométrie plus fine que les points B1, B2 et B3, qui ont subi seulement 22 submersions.

Ainsi, les solides prélevés dans les zones de sédimentation préférentielle ont des caractéristiques granulométriques proches de celles des autres bassins de retenue. Cela est particulièrement vrai pour l'échantillon D1 dont la granulométrie est comparable à celle des dépôts de Périnot (tabl. 3).

\subsubsection{Paramètres de pollution}

Les concentrations en métaux lourds ( $\mathrm{Pb}, \mathrm{Cu}, \mathrm{Cd}$ et $\mathrm{Zn})$, et en hydrocarbures totaux ont été mesurées sur chacun des prélèvements de sédiment réalisés (fig. 6).

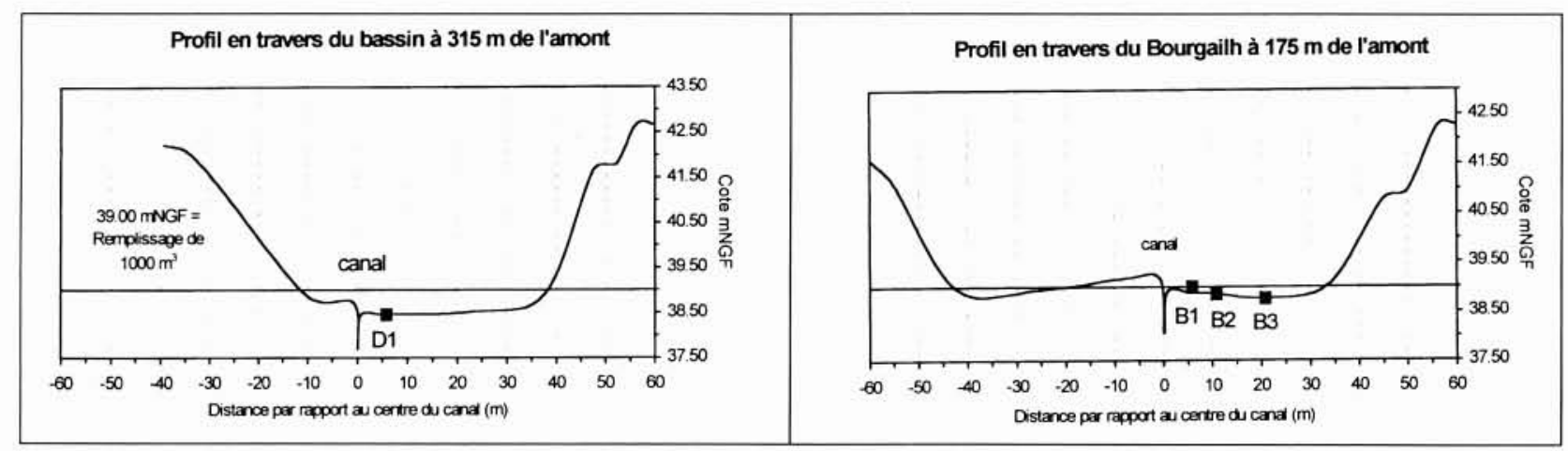

4. Profils en travers du bassin du Bourgailh au niveau des carottages des séries B et D.

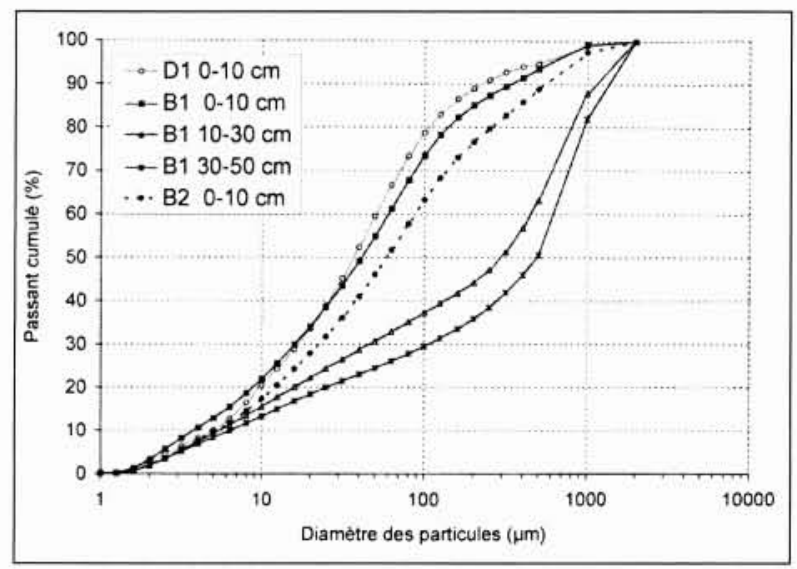

Tableau 3. - Caractéristiques granulométriques et teneur en matières volatiles (MVS) des 3 couches de sol étudiées et de l'échantillon D1 [5].

\begin{tabular}{|l|c|c|c|c|c|}
\hline $\begin{array}{l}\text { Couche/ } \\
\text { Echantillon }\end{array}$ & $\begin{array}{c}\text { Teneur } \\
\text { en } \\
\text { sable } \\
(\%)\end{array}$ & $\begin{array}{c}\text { Teneur } \\
\text { en } \\
\text { limons } \\
(\%)\end{array}$ & $\begin{array}{c}\text { Fraction } \\
<100 \\
\mu m \\
(\%)\end{array}$ & $\begin{array}{c}\mathrm{d}_{50} \\
\mu \mathrm{m} \\
(\%)\end{array}$ & $\begin{array}{c}\text { MVS } \\
(\% \\
\mathrm{MS})\end{array}$ \\
\hline D1 $(0-10 \mathrm{~cm})$ & 41 & 57 & 79 & 67 & 20 \\
Surface $(0-10 \mathrm{~cm})$ & 54 & 45 & 62 & 78 & 16 \\
Moyenne $(10-30 \mathrm{~cm})$ & 62 & 35 & 44 & 234 & 2 \\
Profonde $(30-50 \mathrm{~cm})$ & 64 & 33 & 41 & 341 & 2 \\
\hline
\end{tabular}

5. Courbes granulométriques de sédiments prélevés dans le bassin du Bourgailh.
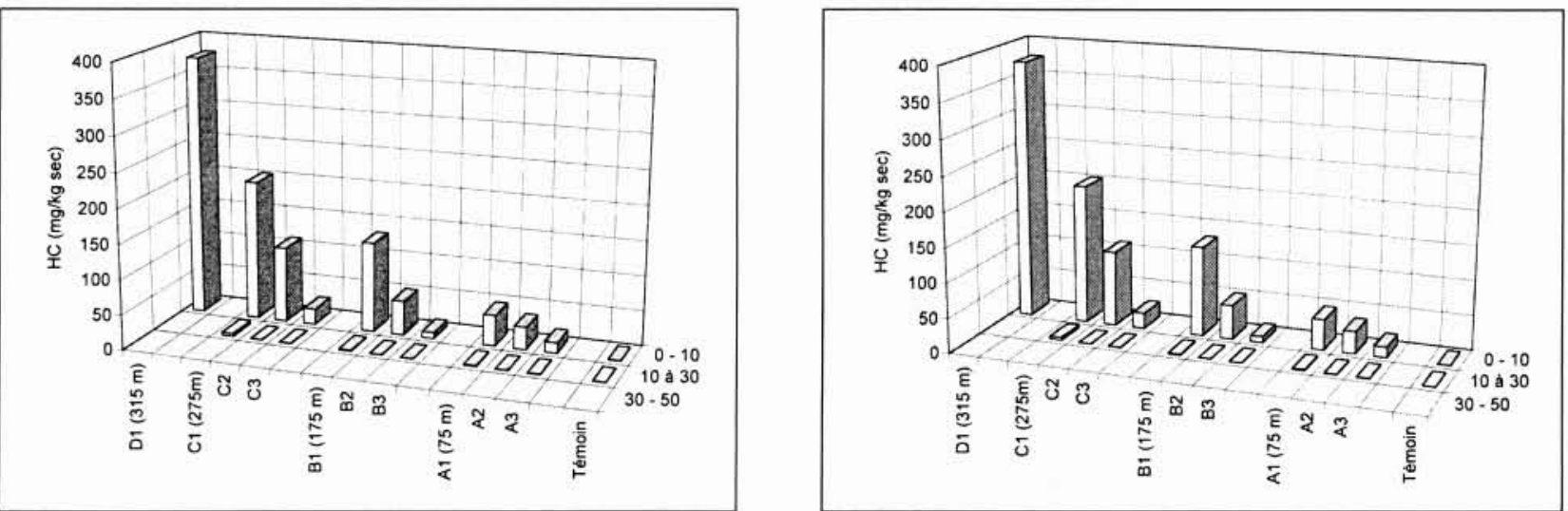

6. Teneurs en hydrocarbures et en cuivre des 11 prélèvements effectués dans le bassin du Bourgailh. 
L'examen des valeurs obtenues fait apparaître une contamination modérée des couches moyenne et profonde ; les teneurs en métaux lourds et hydrocarbures diminuent en profondeur jusqu'à atteindre des valeurs couramment rencontrées dans les sols naturels. En revanche, la couche de surface $(0-10 \mathrm{~cm})$ est en moyenne 5 fois plus chargée en métaux lourds et sa concentration moyenne en hydrocarbures est de $99 \mathrm{mg} / \mathrm{kg}$ sec contre $1,1 \mathrm{mg} / \mathrm{kg} \mathrm{sec}$ pour les couches $10-30 \mathrm{~cm}$ et $30-50 \mathrm{~cm}$.

Tout en restant globalement moins contaminés que les dépôts d'autres bassins de retenue, Périnot notamment, il faut noter, en surface du bassin, les teneurs particulièrement élevées en polluants des points D1, C1 et B1. Comme pour la granulométrie, une dépendance entre les cotes NGF des points de prélèvements, la fréquence de remplissage et les concentrations en polluants ressort assez nettement de la figure 5 (sauf pour la série A). Ainsi, les points qui ont été soumis au plus grand nombre de submersions sont aussi ceux qui sont les plus contaminés.

\section{$\mathrm{V} \square \mathrm{CONCLUSIONS}$}

De l'étude des caractéristiques granulométriques et physicochimiques des dépôts prélevés dans le bassin de Périnot émergent déjà un certain nombre d'idées et d'hypothèses. Ainsi, pendant les phases de stockage des RUTP, une décantation des particules s'opère comme en témoignent les quantités importantes de dépôts dans le bassin et les solides récupérés dans les pièges. Les dispositifs constructifs destinés à l'autocurage du bassin (pente, murets,...) s'avèrent donc être peu efficaces.

Quant aux résultats des analyses granulométriques et des mesures en polluants des sédiments du bassin du Bourgailh, ils tendent à montrer que les prélèvements réalisés en surface, et particulièrement dans la zone aval du bassin, sont constitués de sol naturel et de dépôts issus de la décantation des eaux pluviales pendant les phases de stockage.

Pour progresser dans la connaissance de la caractérisation et la décantation dans les bassins de retenue des solides véhiculés dans les RUTP, une modélisation du fonctionnement hydraulique des bassins est maintenant nécessaire. Pour le bassin de Périnot, elle nous permettra en particulier de déterminer la distribution des champs de vitesse dans le bassin et d'évaluer le phénomène de remise en suspension des solides. Dans le bassin du Bourgailh, le modèle aura pour objet principal de simuler de nouvelles règles de gestion du bassin lors d'événements pluvieux et d'en estimer l'impact sur le rendement de décantation et sur l'augmentation des risques d'inondations.

\section{REMERCIEMENTS}

Cette étude est réalisée avec la participation et le financement du GARIH, de la Communauté Urbaine de Bordeaux, l'Agence de l'Eau Adour-Garonne et du Conseil Régional d'Aquitaine.

\section{BIBLIOGRAPHIE}

[1] BaLAdes J.-D. (1993), - Les solutions compensatoires en assainissement urbain. Formation continue de l'ENGEES, Strasbourg, juin 1993, $21 \mathrm{p}$.

[2] Bertrand-Krajewski J.-L. (1993). - Pollution des rejets urbains de temps de pluie. Synthèse générale. Rapport CIRSEE Lyonnaise des Eaux-Dumez, n ER.ABE.94.03, novembre 1993. $137 \mathrm{p}$.

[3] Cheвво G. (1992), - Solides des rejets urbains de temps de pluie : caractérisation et traitabilité. Thèse de doctorat de l'ENPC, Paris, mars 1992, $413 \mathrm{p}$.

[4] Jacopin C., Bertrand-Krajewski J.-L., Desbordes M. (1997a). - Présentation des premières campagnes d'étude de la décantation dans le bassin de retenue de Périnot. Rapport d'avancement GARIH - Lyonnaise des Eaux, février 1997, $43 \mathrm{p}$

[5] Jacopin C., Bertrand-Krajewski J.-L., Legret M. (1997b). — Présentation des premières campagnes d'étude de la décantation dans le bassin de retenue du Bourgailh. Rapport d'avancement GARIH - Lyonnaise des Eaux, février 1997. $29 \mathrm{p}$ 